\title{
INTEGRAL FUNCTIONS OBTAINED BY COMPOUNDING POLYNOMIALS*
}

BY J. F. RITT

1. Introduction. We consider a sequence of polynomials $P_{n}(z),(n=1,2, \cdots)$, where the degrees of the $P_{n}$ do not exceed a fixed integer $m$ and where each $P_{n}$, ordered in ascending powers of $z$, starts with the term $z$. We shall study the sequence of polynomials $Q_{n}(z)$ defined by

$$
Q_{1}(z)=P_{1}(z) ; Q_{n+1}(z)=Q_{n}\left[P_{n+1}(z)\right], \quad(n=1,2, \cdots),
$$

and also the sequence of polynomials $R_{n}(z)$ defined by

$$
R_{1}(z)=P_{1}(z) ; R_{n+1}(z)=P_{n+1}\left[R_{n}(z)\right], \quad(n=1,2, \cdots) .
$$

If the coefficients, after the first, in $P_{n}$, are sufficiently small, these sequences will converge to integral functions. For instance, $\sin z$ can be obtained, in many ways, as a limit of a sequence (1). In what follows, our chief object will be to establish conditions under which the sequences converge to integral functions.

2. The Sequence of $Q_{n}(z)$. Let

$$
P_{n}(z)=z+a_{n 2} z^{2}+\cdots+a_{n m} z^{m}, \quad(n=1,2, \cdots),
$$

where $m$ is an integer independent of $n$.

THEOREM 1. Let a convergent series of positive numbers,

$$
c_{1}+c_{2}+\cdots+c_{n}+\cdots,
$$

exist such that $\left|a_{n i}\right|<c_{n}$, for every $n$ and for $i=2, \cdots, m$. Then the sequence of polynomials $Q_{n}(z)$ converges to an integral function, the convergence being uniform in every bounded domain.

Proof. For every $n$,

$$
U_{n}(z)=z+c_{n}\left(z^{2}+\cdots+z^{m}\right)
$$

is a majorant of $P_{n}(z)$. Let

$$
V_{1}=U_{1} ; V_{n+1}=V_{n}\left(U_{n+1}\right), \quad(n=1,2, \cdots) .
$$

\footnotetext{
* Presented to the Society, April 14, 1933.
} 
Then $V_{n}$ is a majorant of $Q_{n}$. Also, if we let

we have

$$
\alpha_{n}=c_{n}\left(z^{2}+\cdots+z^{m}\right),
$$

$$
\begin{aligned}
V_{n+1}-V_{n} & =V_{n}\left(z+\alpha_{n+1}\right)-V_{n} \\
& =\frac{d V_{n}}{d z} \alpha_{n+1}+\frac{1}{2 !} \frac{d^{2} V_{n}}{d z^{2}} \alpha_{n+1}^{2}+\cdots,
\end{aligned}
$$

from which it follows easily that $V_{n+1}-V_{n}$ is a majorant of $Q_{n+1}-Q_{n}$. For every positive $z, V_{n+1}(z)>V_{n}(z)$. These considerations show that our theorem will be proved if we can show that the sequence of $V_{n}$ converges for every positive $z$.

Let $b$ be any positive number. Let

$$
h=2 b+4 b^{2}+\cdots+2^{m-1} b^{m-1} .
$$

Then the infinite product $\left(1+h c_{1}\right) \cdots\left(1+h c_{n}\right) \cdots$ converges. Let $p$ be a fixed integer such that

$$
\left(1+h c_{p+1}\right)\left(1+h c_{p+2}\right) \cdots<2 .
$$

Let

$$
W_{1}=U_{p+1} ; W_{n+1}=W_{n}\left(U_{p+n+1}\right), \quad(n=1,2, \cdots) .
$$

It will plainly suffice to show that the sequence of $W_{n}$ converges for $z=b$. For any $n$, by (4) and (5),

$$
U_{p+n}(b)<b\left(1+h c_{p+n}\right),
$$

so that, by $(6), U_{p+n}(b)<2 b$. Hence

$$
\begin{aligned}
U_{p+n-1}\left[U_{p+n}(b)\right] & =U_{p+n}(b)\left[1+c_{p+n-1}\left(U_{p+n}(b)+\cdots\right)\right] \\
& <U_{p+n}(b)\left[1+h c_{p+n-1}\right] \\
& <b\left(1+h c_{p+n-1}\right)\left(1+h c_{p+n}\right),
\end{aligned}
$$

and the last quantity, by (6), is less than $2 b$. Continuing in this fashion, we find that, for every $n$,

$$
W_{n}<b\left(1+h c_{p+1}\right) \cdots\left(1+h c_{p+n}\right)<2 b .
$$

This shows that the $W_{n}(b)$, which increase with $n$, approach a limit. The theorem is proved.

That the condition placed on the $P_{n}$ is critical with respect to the convergence of the $Q_{n}$, is seen on taking $P_{n}=z+c_{n} z^{m}$ with 
$c_{n}>0$ and (3) divergent. The coefficient of $z^{m}$ in $Q$ will be $c_{1}+\cdots+c_{n}$ and $Q_{n}$ will tend towards infinity with $n$ for every positive $z$.

The function $\sin z$ can be expressed as a limit of polynomials $Q_{n}$. Let

$$
P_{n}(z)=z-\frac{4}{3^{2 n+1}} z^{3} .
$$

The formula

$$
\sin z=3 \sin \frac{z}{3}-4 \sin ^{3} \frac{z}{3}
$$

gives then

$$
\sin z=Q_{n}\left(3^{n} \sin 3^{-n} z\right) .
$$

From (7) we see that the $Q_{n}$ converge to an integral function. This integral function must be $\sin z$, since $3^{n} \sin 3^{-n} z$ approaches $z$ as $n$ increases.* $^{*}$

3. The Sequence of $R_{n}(z)$. We shall study the sequence of $R_{n}(z)$ defined by (2).

Theorem 2. Let the $P_{n}(z)$ all be of degree at most $m>1$. Let a sequence of positive numbers $c_{n}$ exist such that

$$
\limsup _{n \rightarrow \infty} c_{n}^{1 / m^{n}}<1
$$

and'such that, for every $n$, the moduli of the coefficients of $z^{2}, \cdots, z^{m}$ in $P_{n}$ are all less than $c_{n}$. Then the $R_{n}(z)$ converge to an integral function, the convergence being uniform in every bounded domain.

Proof. Let $r$ be a number which lies between the two members of (8). Then, for $n$ large,

$$
z+r^{m^{n}}\left(z^{2}+\cdots+z^{m}\right)
$$

will be a majorant of $P_{n}(z)$. A fortiori, since $m>1$,

$$
U_{n}(z)=z+r^{m n-1} z^{2}+r^{2 m^{n-1}} z^{3}+\cdots+r^{(m-1) m^{n-1} z^{m}}
$$

* In the same way, one can express as limits of polynomials $Q_{n}$ a large class of the functions with rational multiplication theorems introduced by Poincaré (Journal de Mathématiques, vol. 55 (1890)). 
will be a majorant of $P_{n}(z)$ for $n$ large. We see now readily that it will suffice, for the proof of our theorem, to show that the sequence of $V_{n}(z)$ defined by

$$
V_{1}=U_{1} ; V_{n+1}=U_{n+1}\left(V_{n}\right),
$$$$
(n \geqq 1) \text {, }
$$

converges for every real and positive $z . \dagger$

Let $p$ be any non-negative integer. Putting

$$
W_{1}=U_{p+1} ; W_{n+1}=U_{p+n+1}\left(W_{n}\right), \quad(n \geqq 1),
$$

we shall show that the sequence of $W_{n}$ converges for $z<h r^{-m^{p}}$, where $h=1-r$.

By (9),

$$
S_{n}(z)=\frac{z}{1-r^{m p+n-1} z}, \quad(n=1,2, \cdots),
$$

is a majorant of $U_{p+n}$. If, then,

$$
T_{1}=S_{1} ; T_{n+1}=S_{n+1}\left(T_{n}\right), \quad(n \geqq 1),
$$

$T_{n}$ will be a majorant of $W_{n}$. Now an easy calculation shows that

$$
T_{n}(z)=\frac{z}{1-\left(r^{m p}+\cdots+r^{m p+n-1}\right) z} .
$$

For any positive $z$ less than the reciprocal of the infinite series

$$
r^{m p}+r^{m p+1}+\cdots,
$$

which reciprocal we shall denote by $k$, the $T_{n}(z)$ form a sequence of numbers which increase towards $k z /(k-z)$. Also, if $0<z<k, T_{n}(z)>W_{n}(z)$, so that the $W_{n}(z)$ will form a bounded sequence of increasing numbers and will converge to a limit. Now as $m>1$,

$$
k \geqq \frac{r^{-m p}}{1+r+r^{2}+\cdots}=h r^{-m p},
$$

and our statement with respect to (11) is proved.

Thus Theorem 2 will be established if, putting $V_{0}(z)=z$, we show that for every positive $z$ there is a $p$ such that $V_{p}(z)<h r^{-m^{p}}$.

$\dagger$ The fact that $U_{n}$ may not be a majorant of $P_{n}$ for $n$ small is of no significance. One may suppress a finite number of $P_{n}$ and then add a finite number of polynomials (9) to the beginning of the resulting sequence of $U_{n}$. 
Let us assume that there is a positive $z$ for which no such $p$ exists. In what follows, we work with a fixed $z$ of this type. We have, by (9) and (10), for any $n \geqq 0$,

$$
\begin{aligned}
V_{n+1} & =V_{n}+r^{m n} V_{n}^{2}+\cdots+r^{(m-1) m^{n}} V_{n}^{m} \\
& \leqq V_{n}\left(1+r^{m n} V_{n}\right)^{m-1} .
\end{aligned}
$$

Now, for every $n$,

$$
V_{n} \geqq h r^{-m^{n}},
$$

so that

$$
1 \leqq \frac{r^{m^{n}} V_{n}}{h},
$$

and, if we put $a=(1+1 / h)^{m-1}$, we have, by (12),

$$
V_{n+1} \leqq a r^{(m-1) m^{n}} V_{n}^{m} \leqq a r^{m^{n}} V_{n}^{m} .
$$

We have thus

$$
V_{1} \leqq a r z^{m}, V_{2} \leqq a^{m+1} r^{2 m_{z} m^{2}}, V_{3} \leqq a^{m 2+m+1} r^{3 m^{2}} z^{m^{3}},
$$

and, in general,

$$
V_{n+1} \leqq a^{m^{n+\cdots+1}} r^{(n+1) m^{n}} z^{m^{n+1}} .
$$

As $m>1$, we have $m^{n}+\cdots+1<m^{n+1}$. Then, because $a>1$,

$$
V_{n+1}<\left[r^{(n+1) / m} a z\right]^{m^{n+1}} .
$$

As $z$ is fixed, $r^{(n+1) / m} a z$ is small for $n$ large, so that, by (14), $V_{n+1}$ approaches 0 as $n$ increases. This contradicts (13). The theorem is proved.

The condition (8) is a critical one. That we cannot let the first member of (8) be as great as unity is seen on taking $P_{n}=z+z^{m}$. The coefficient of $z^{m}$ in $Q_{n}$ will be $n$ and the $Q_{n}$ will diverge for every positive $z$. That $m$ in the first member of (8) cannot be replaced by any smaller positive number $\alpha$, is seen, taking $m=2$, for instance, on putting $P_{n}=z+2^{-\alpha^{n}} z^{2}$. For any positive $z$, we have

$$
P_{n}>2^{-\alpha^{n}} z^{2} \text {. }
$$

Then

$$
R_{1}>2^{-\alpha} z^{2}, R_{2}>2^{-\left(\alpha^{2}+2 \alpha\right)} z^{4},
$$


and, in general,

$$
R_{n}>2^{-\left(\alpha^{n}+2 \alpha^{n-1}+\cdots+2^{n-1} \alpha\right)} z^{2^{n}} .
$$

Now

$$
-\left(\alpha^{n}+2 \alpha^{n-1}+\cdots+2^{n-1} \alpha\right)=\frac{\alpha^{n+1}-2^{n} \alpha}{2-\alpha}>-b 2^{n},
$$

where $b=\alpha /(2-\alpha)$. Thus

$$
R_{n}>\left(\frac{z}{2^{b}}\right)^{2^{n}},
$$

so that the $R_{n}$ diverge for $z>2^{b}$.

Let $f(z)$ be an integral function obtained as a limit of polynomials $R_{n}(z)$, the approach being uniform in every bounded domain. Unless $P_{n}(z)=z$ for every $n, f(z)$ will not be linear, for if some $R_{n}(z)$ is of degree greater than unity, $f(z)$, like that $R_{n}(z)$, will assume certain values at more than one place. In what follows, we shall assume that $f(z)$ is not linear.

We are going to prove that, between any two branches of the inverse of $f(z)$, there exists an algebraic relation of a simple type.

Let $a$ and $b$ be two distinct points such that $f(a)=f(b)$ and that the derivative of $f(z)$ does not vanish at $a$ or at $b$. Let $A$ be a circle with $a$ as center such that, in the interior of $A, f(z)$ assumes no value twice. Let $B$ be a similar circle with center at $B$. We can find a neighborhood $M$ of $f(a)=f(b)$ such that, both in $A$ and in $B, R_{n}(z)$ with $n$ large assumes all values in $M$. If $n$ is large enough, $R_{n}(a)$ will be in $M$. In what follows, we deal with a fixed $R_{n}(z)$ for which both conditions just described are realized.

If $z_{a}$ is a point in $A$, very close to $a$, there will be a $z_{b}$ in $B$ such that $f\left(z_{b}\right)=f\left(z_{a}\right)$, and, furthermore, $R_{n}\left(z_{a}\right)$ will lie in $M$. We shall prove that $R_{n}\left(z_{a}\right)=R_{n}\left(z_{b}\right)$. As $R_{n}\left(z_{a}\right)$ is in $M$, there is a $\zeta$ in $B$ such that $R_{n}(\zeta)=R_{n}\left(z_{a}\right)$. Now $\zeta$ must coincide with $z_{b}$, for $f(\zeta)=f\left(z_{a}\right)=f\left(z_{b}\right)$ and $f(z)$ assumes no value twice in $B$.

Thus, if we put $w=f(z)$ and if $\alpha(w)$ and $\beta(w)$ are two branches of the inverse of $f(z)$, then, for $n$ large, $R_{n}[\alpha(w)]=R_{n}[\beta(w)]$.

Columbia University 